\title{
Applying CRITOE in pediatric elbow trauma: is that a fracture or an ossification center?
}

\author{
Jack G. Underschultz ${ }^{1}$. Andrew Stagg ${ }^{2} \cdot$ Cameron K. MacGougan ${ }^{2}(\mathbb{0}$
}

Received: 29 October 2020 / Accepted: 23 December 2020 / Published online: 15 February 2021

(C) The Author(s), under exclusive licence to Canadian Association of Emergency Physicians (CAEP)/ Association Canadienne de Médecine d'Urgence (ACMU) 2021

Keywords Medial epicondyle $\cdot$ Elbow $\cdot$ Fracture $\cdot$ X-ray $\cdot$ Pediatrics

A 5-year-old girl presented to the Emergency Department after falling off a dryer onto a cement floor with her left elbow being the primary point of contact. There was significant swelling, bruising, and tenderness to the medial elbow joint but no obvious deformity. The skin was intact and her compartments were soft. Her distal neuro-vascular exam was normal.

Anteroposterior, lateral, and internal oblique radiograph views of the left elbow were obtained. (Fig. 1). What is your interpretation?

One approach to the pediatric elbow radiograph interpretation is to use AABC'S-adequacy, alignment, bone, cartilage, and soft tissue [1, 2]:

Adequacy ensure the lateral X-ray is a true lateral by looking for an hour-glass appearance of the distal humerus.

Alignment the anterior humeral line, drawn down the anterior surface of the humerus, should project through the middle third of the capitellum. The radiocapitellar line, drawn along the radial neck, should always project through the capitellum on the AP and lateral views.

Bone assess for disruption of the cortex with every bone. Assess your ossification centers.

Cartilage examine the joint spaces for widening.

Soft tissue presence of an anterior fat pad may be a normal finding. Presence of a posterior fat pad indicates an effusion.

Staff radiology documented the presence of a joint effusion but did not comment on any additional abnormalities.

Cameron K. MacGougan

ckm@ualberta.ca

1 University of Alberta, Edmonton, AB, Canada

2 Department of Emergency Medicine, University of Alberta, 790 University Terrace Building, 8303-112 Street, Edmonton, AB T6G 2T4, Canada
On closer examination by the staff emergency physician, a fragment was noticed in the anatomical position of the trochlea. This was abnormal as the typical age of trochlear ossification is around 7 years of age. More important, the medial epicondyle ossification center, which reliably ossifies before the trochlea, was absent. This led to a diagnosis of a significantly displaced medial epicondyle avulsion fracture which was confirmed with a comparison view of the right elbow demonstrating the medial epicondyle in its anatomical position and an absent trochlea ossification center.

Operative treatment was done by closed reduction of the medial epicondyle fracture under fluoroscopic guidance followed by cast immobilization. The patient recovered uneventfully.

Medial epicondyle fractures of the humerus account for $5-10 \%$ of pediatric elbow fractures, with a peak incidence between 9 and 14 years of age [3]. The injury occurs from a forceful valgus strain on the joint with approximately $50 \%$ of cases associated with elbow dislocation [3]. Computed tomography (CT) scans are most sensitive for diagnosing and accurately measuring displacement of the fracture [4]. However, due to associated cost and radiation burden, radiographs are most commonly obtained with internal oblique and distal humerus axial views being most sensitive [4]. The differential diagnoses for a medial epicondyle fracture include osteochondrosis of the medial epicondyle and fracture of the medial condyle.

Order of appearance of the elbow ossification centers is very reliable in most individuals and helpful in proper diagnosis of traumatic pediatric elbow injuries. The order of chronological appearance is described commonly by the acronym "CRITOE": (C)apitellum, (R)adial head, (I)nternal (or medial) epicondyle, (T)rochlea, (O)lecranon, (E)xternal (or lateral) epicondyle [2]. The age at which the ossification centers appear is more variable; however, there are two 

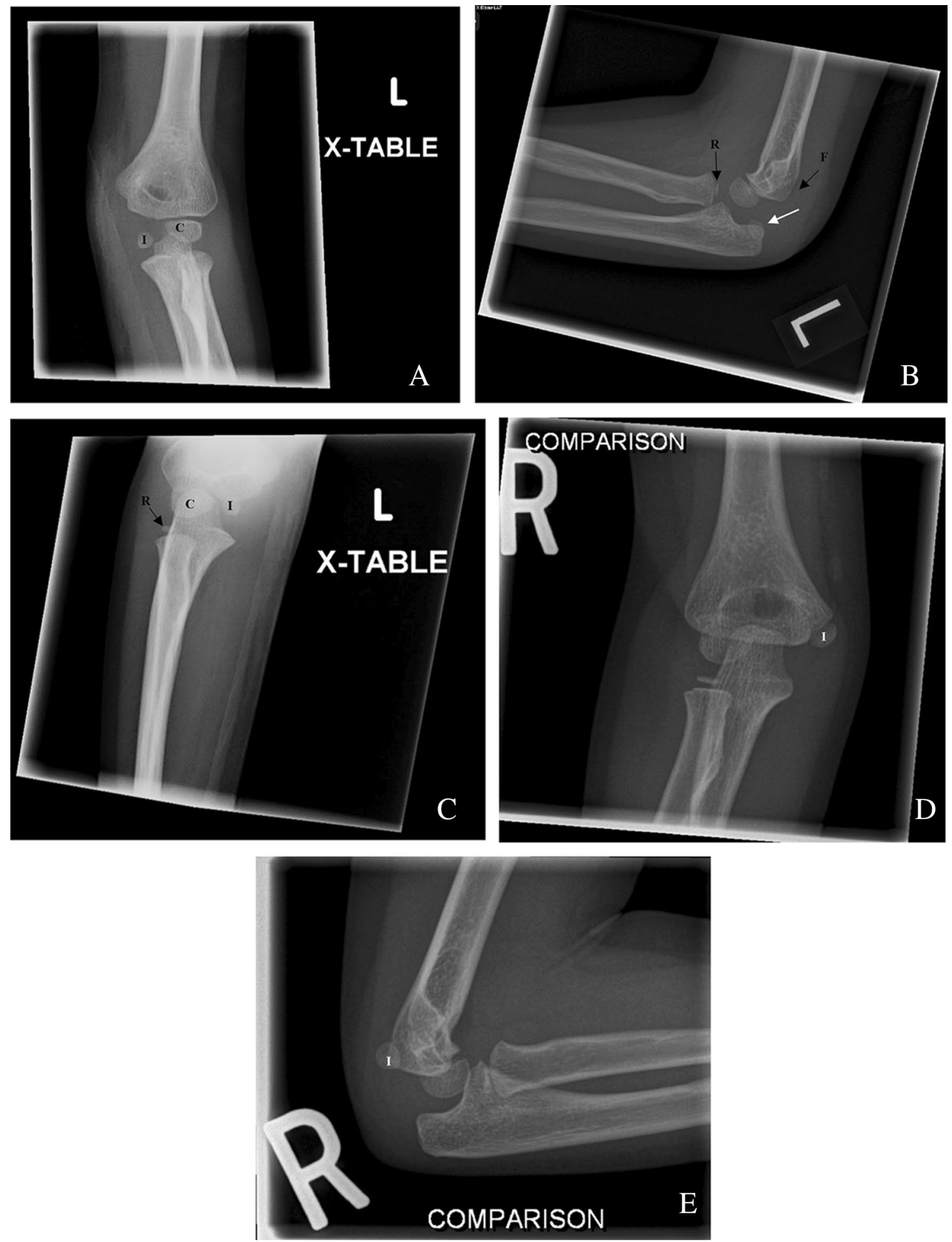

Fig. 1 a Anteroposterior view with markings. This identifies the ossification centers of the capitulum (C) and the displaced internal (medial) epicondyle (I). The radial head is difficult to visualize in this view. b Lateral view with markings. This shows the ossification center of the radial head ( $\mathrm{R}$ with black arrow), posterior fat pad ( $\mathrm{F}$ with black arrow) and the displaced medial epicondyle (white arrow). c Internal oblique view with markings. This identifies the ossifica- tion centers of the capitulum (C), radial head ( $\mathrm{R}$ with arrow) and the displaced internal (medial) epicondyle (I). d Contralateral anteroposterior view with the internal (medial) epicondyle (I) ossification in its normal anatomical position. e Contralateral lateral view with the internal (medial) epicondyle (I) ossification in its normal anatomical position 
counting methods taught: the simplified version of $1,3,5,7$, 9 , and 11 years of age, respectively; and the more accurate $1,5,7,10,10$, and 11 years of age [1].

For older children, the order of fusion of the ossification centers, which is different than order of appearance, can be equally important in making a diagnosis of a medial epicondyle fracture. Here, the order of fusion is "CTE-RO-I": the capitulum, trochlea, and external (lateral) epicondyle fuse together around age 13 , then fuse to the distal humerus around age 15 [4]. The radius, olecranon, and internal (medial) epicondyle fuse around ages 15, 16, and 17, respectively [4]. Focusing on the pattern of ossification appearance and fusion is paramount for not mistaking a displaced medial epicondyle fracture for apparent normal orthopedic anatomy. Delayed diagnosis and mismanagement are associated with poor prognosis and high complication rates [5]. All medial epicondyle fractures should be seen by an orthopedic surgeon. Uncomplicated fractures can be managed conservatively with casting; however, surgical treatment with internal fixation is required with complicated cases such as significant displacement or ulnar neuropathy [5]. With appropriate management, recovery of full function is expected [5].

\section{Compliance with ethical standards}

Conflict of interest The authors, Jack Underschultz, Andrew Stagg and Cameron MacGougan, have no relevant financial or non-financial interests to disclose.

\section{References}

1. Weerakkody Y, Jones J. Pediatric elbow radiograph (an approach). Radiopaedia. 2020. https://radiopaedia.org/articles/paediatric -elbow-radiograph-an-approach. Accessed 14 Oct 2020.

2. Sayal A. How to avoid missing a pediatric elbow fracture. ACEP Now. 2020. https://www.acepnow.com/article/how-to-avoid-missi ng-a-pediatric-elbow-fracture/. Accessed 14 Oct 2020.

3. Wheeless III CR. Wheeless' textbook of orthopaedics: medial epicondyle frx of the humerus. 2015. http://www.wheelessonline. com/ortho/medial_epicondyle_frx_of_the_humerus. Accessed 14 Oct 2020.

4. Patel B, Reed M, Patel S. Gender-specific pattern differences of the ossification centers in the pediatric elbow. PediatrRadiol. 2009;39:226-31. https://doi.org/10.1007/s00247-008-1078.-4.

5. Lee HH, Shen HC, Chang JH, Lee CH, Wu SS. Operative treatment of displaced medial epicondyle fractures in children and adolescents. J Shoulder Elbow Surg. 2005;14(2):178-85. https:// doi.org/10.1016/j.jse.2004.07.007. 